Research Article

Open Access

\title{
A Prospective study on Principles and Outcomes of Reconstructive Flaps in Carcinoma of Cheek
}

\author{
Vinay $\mathbf{G}^{\mathbf{1}}$, Mohan $\mathbf{G}^{2}$, Balasubrahmanya KS $\mathbf{1}$ and Prakash SS 3 \\ IDepartment of General Surgery, Mysore Medical College and Research Institute, Mysore, Karnataka, India \\ 2 Department of Plastic Surgery, Mysore Medical College and Research Institute, Mysore, Karnataka, India \\ ${ }^{3}$ Department of Surgical Oncology, Mysore Medical College and Research Institute, Mysore, Karnataka, India
}

\section{Article Info}

\section{*Corresponding author: \\ Vinay $\mathbf{G}$}

Department of General Surgery

Mysore Medical College and Research Institute Mysore, Karnataka,

India

Tel: +91 9986929929

E-mail: dr.vinay1990@gmail.com

\section{Received: January 13, 2018 \\ Accepted: January 27, 2018 \\ Published: February 01, 2018}

Citation:Vinay G, Mohan G, Balasubrahmanya KS, Prakash SS. A Prospective study on Principles and Outcomes of Reconstructive Flaps in Carcinoma of Cheek. Madridge J Surg. 2018; 1(1): 14-18.

doi: 10.18689/mjs-1000104

Copyright: () 2018 The Author(s). This work is licensed under a Creative Commons Attribution 4.0 International License, which permits unrestricted use, distribution, and reproduction in any medium, provided the original work is properly cited.

Published by Madridge Publishers

\begin{abstract}
Background: Cancers of the oral cavity present in a variable way but are often associated with persisting swelling or ulceration within the oral cavity. In the Indian subcontinent, oropharyngeal cancer is the common malignant tumour accounting for $40 \%$ of all cancers.
\end{abstract}

Aims and Objectives: To study the principles and outcomes of flaps used in reconstruction of carcinoma of cheek in view of maintaining oral competence, facilitate swallowing, preserving speech, prevent aspiration and to achieve better cosmesis.

Materials and Methods: A prospective, open label, randomized, hospital based, single centered study was conducted among 60 subjects attending Surgical Oncology OPD, K.R. Hospital attached to Mysore Medical College And Research Institute, Mysore meeting the inclusion and exclusion criteria over a period of 24 months from January 2016 to December 2017. All patients who present with symptoms and signs of Carcinoma cheek, and confirmed by Edge Biopsy, FNAC of Nodes, X-Ray mandible and ortho pantamogram were included in the study. Patients suffering from Ca lip, Ca tongue, Ca oropharynx and Ca maxilla are excluded. Descriptive statistics, Chi-square test, Multivariate Cox regression analysis were used to analyse the results.

Results: The mean age group of the study subjects was $52.63 \pm 7.48$ (34-86) years. The gender distribution showed $52(86.67 \%)$ females as compared to $8(13.33 \%)$ males. Maximum number of $29(48.33 \%)$ patients presents in Stage-IV while minimum number of $8(13.33 \%)$ patients presented in Stage-II. $13(21.67 \%)$ and $10(16.67 \%)$ patients presented in Stage-III and Stage-I respectively. 15(25\%) and 17(28.33\%) of patients underwent reconstruction with the Pectoralis major myocutaneous flap(PMMC) \& PMMC+DP Flaps respectively. 6(10\%) patients underwent tongue flap while $5(8.33 \%)$ patients underwent forehead flap ( $p<0.0001) .4(6.67 \%)$ and $1(1.67 \%)$ patients underwent Deltopectoral (DP) and tongue + DP flap respectively. Postoperative complications like infection and necrosis occurred in $3(5 \%)$ and $1(1.67 \%)$ cases respectively, while orocutaneous fistula occurred in $1(1.67 \%)$ cases and $1(1.67 \%)$ case showed recurrence.

Conclusion: Patients subjected to reconstruction of cheek defect with PMMC \& PMMC + DP flap shows better results in terms of oral competence, speech, swallowing \& cosmetic appearance when compared to other flaps.

Keywords: Carcinoma of cheek; Cosmesis; Flaps; Oral competence; Reconstruction of cheek.

\section{Introduction}

Cancers of the oral cavity present in a variable way but are often associated with persisting swelling or ulceration within the oral cavity. In the western world oral or oropharyngeal cancer is rare, accounting for $2-4 \%$ of all malignant tumors, although there is evidence that the incidence is on the increase, particularly among young people. 
In the Indian subcontinent, however, oropharyngeal cancer is the common malignant tumor accounting for $40 \%$ of all cancers [1].

Approximately 13,500 cases of carcinoma buccal mucosa have been reported from the various Indian cancer registries during 1990-96 [2,3] in comparison to 1272 cases registered worldwide during this six year period. The high incidence of carcinoma buccal mucosa in India is probably attributable to usage of tobacco in its various forms and smoking. Among the 8000 cases reported in Indian hospital based cancer registries during 1994-98, locally advanced cancers accounted for 70\% [4]. The reported 5 year survival rates for buccal mucosa cancers in India ranges from $80 \%$ for stage I disease to $5-15 \%$ for locally advanced disease $[5,6]$. Most of these lesions present in a locally advanced stage requiring curative or palliative treatment as first line of management. This results in significant quantity of soft tissues loss which requires reconstruction. Tobacco was identified as the causative agent in as many as of the patients, majority of whom presented in the 51-60 age groups a non-healing ulcer. Surgical management consists of wide local excision with local or distant flap reconstruction with neck dissection. Majority of the cases were in stage-IV with recurrence rate is 3.3\%. Complication occurred in around $80 \%$ of the cases. Due to lack of health consciousness, low socio-economic status, habit of chewing paan and tobacco in Indian population [2], inspite of easy accessibly to early diagnosis the number of locally advanced lesions are seen more due to delay in presentation. It is a well established fact that surgery is the first line of treatment in locally advanced tumors of buccal mucosa (P.H. O'Brein \& D. Cathin, 1965 and Bloom \& Spiro 1980) [7]. Advances in radiotherapy and chemotherapy have undoubtedly increased its value handling many lesions of the buccal mucosa. However, for advanced lesion of buccal mucosa involving lymph nodes, surgical excision with reconstruction, remains the treatment of choice [8]. Surgery enables the surgeon to assess accurately the extent of spread of the growth, to undertake the simultaneous resection of nearby involved tissue and to judge the prognosis. Metastatic cervical lymph nodes are best taken care of by surgery. Surgical dissection can remove all important nodes. Combined modality of surgery, followed by post-operative radiation therapy remains the mainstay of the treatment for obtaining a good survival rate [8]. The aim of radical surgery is to remove all the disease. Once the decision to operate has been made, consideration of cosmesis and functional difficulties must not be allowed to compromise the extent of resection. This has been possible with the development of excellent reconstruction techniques.

On the basis of anatomical content of flaps and the nature of their vascular patterns flaps can be categorised into fasciocutaneous, muscle and myocutaneous, and free flaps. Fasciocutaneous flaps rely entirely on the pattern of blood vessels running horizontally in the superficial fascia, reinforced in many body sites by the rich plexus of small vessels in relation to the general investing layer of deep fascia. With this as their vascular background, three categories of flaps include; axial pattern flaps, random pattern flaps, and fasciocutaneous flaps has been described. Random pattern flaps have insufficient preexisting bias in the pattern of their arteries and veins $[9,10]$.
The principle of moving a muscle as a flap in myocutaneous flaps is to manage the problem of a surface which is unsuitable for skin grafting is by covering it with the highly vascular muscle belly, on top of which a skin graft can be applied. Alternatively, the graft may be meshed and applied primarily. Skin element can safely rely entirely on the underlying muscle for its blood supply, and it is now generally transferred with the muscle as an island $[9,10]$.

Forehead flap is composed of frontalis muscle from hairline to eyebrows with overlying skin and subcutaneous tissue. It can be used as hemi forehead flap or total forehead flap, blood supply is by superficial temporal artery \& post auricular artery. The advantage is that it is near to oral cavity, hairless or firmly holds the suture very well, blood supply is excellent presence of a longitudinally and horizontally oriented vasculature permits rotation on the radius of either the superficial temporal or supra orbital artery $[9,10]$.

Medially based deltopectoral flap is used by Bakamjian in 1965 for Soft tissue reconstruction of mandible or maxilla but now it is an alternative for PMMC flaps, it is composed of skin, subcutaneous tissue, fascia but no muscle. Blood supply is by internal mammary artery. Advantage is larger amount of skin is available and hairless can be used along with myocutaneous flap to cover mucosal or skin coverage. Oral fistula is common which can be managed conservatively or it is closed at second surgery $[9,10]$.

Pectoralis major myocutaneous flaps are of three types, PMMC Island flap, PMMC Paddle flap and Gemini flap. Advantage is arch of rotation will be more than $20 \mathrm{~cm}$, it is very bulky, functionally or cosmetically it gives good results and there will be early closure of donar area it is an hair less area. Major disadvantage is Loss of muscle is noticeable in male and it is difficult to identify vascular pedicle $[9,10]$.

Tongue flaps are indicated in oro nasal communications and oro antral communications, lip reconstruction, buccal mucosa reconstruction and reconstruction of the hypopharynx. Dorsal tongue flap is classified into posteriorly based flap and anteriorly based flap used in anterior buccal mucosa, posterior buccal mucosa. Flap elevation posterior to circumvallate papilla should be avoided. Lateral tongue flap is indicated in buccal mucosa, lateral palate, oroantral communication. Ventral or dorsal-v shaped fashion flaps helps in primary closure. Double door tongue flap is to reline large defects of the buccal mucosa, extending from the commisure of the anterior mandibular ramus $[9,10]$.

The objective here is to study the principles and outcomes of flaps used in reconstruction of carcinoma of cheek in view of maintaining oral competence, facilitate swallowing, preserving speech, prevent aspiration and to achieve better cosmesis.

\section{Materials and Methods}

A prospective, open label, randomized, hospital based, single centered study was conducted among 60 subjects attending Surgical Oncology OPD, K.R. Hospital attached to Mysore Medical College And Research Institute, Mysore meeting the inclusion and exclusion criteria over a period of 24 months from January 2016 to December 2017. 
All patients who present with symptoms and signs of Carcinoma cheek, and confirmed by Edge Biopsy, FNAC of Nodes, X-Ray mandible and Orthopantamogram were included in the study. Patients suffering from Ca lip, Ca tongue, Ca oropharynx and Ca maxilla are excluded.

Descriptive statistics, Chi-square test, Multivariate Cox regression analysis were used to analyse the results. $p<0.05$ was considered statistically significant. The Statistical software namely SPSS 21.0, Stata 15.1, MedCalc 17.9 and Systat 13.0 were used for the analysis of the data and Microsoft word and Excel have been used to generate graphs and tables.

\section{Results}

During the twelve months study period, all 60 cases underwent surgery \& reconstruction of defect with flaps. The mean age group of the study subjects was $52.63 \pm 7.48$ (34-86) years. $27(45 \%)$ patients of carcinoma cheek seen in age between $50-59$ yrs. Least incidence $4(6.67 \%)$ occurred in age between $70-80 \mathrm{yrs}$. The gender distribution showed $52(86.67 \%)$ females as compared to $8(13.33 \%)$ males $(p<0.001)$.

Highest incidence of carcinoma of cheek seen in 49(81.67\%) of patients chewing tobacco, $4(6.67 \%)$ of patients indulged in tobacco and smoking while $7(11.67 \%)$ patients indulged in tobacco smoking and alcohol. 44(73.33\%) of patients presents with ulcer, swelling with ulcer in cheek in $4(6.67 \%)$ and $12(20 \%)$ presented with trimus and ulcer $(p=0.126)$.

Maximum number of $29(48.33 \%)$ patients presents in Stage-IV while minimum number of $8(13.33 \%)$ patients presented in Stage-II. 13(21.67\%) and 10(16.67\%) patients presented in Stage-III and Stage-I respectively (Table 1 and Graph 1).53(88.33\%) of cases were well differentiated squamous cell carcinoma, $7(11.67 \%)$ cases were moderately differentiated while none of them were poorly differentiated on histopathology $(p=0.674)$.

Table 1. Clinical Stage

\begin{tabular}{|c|c|c|c|}
\hline TNM Stage & Male & Female & Total \\
\hline Stage-I & $1(1.67 \%)$ & $9(15 \%)$ & $10(16.67 \%)$ \\
\hline Stage-II & $2(3.33 \%)$ & $6(10 \%)$ & $8(13.33 \%)$ \\
\hline Stage-III & $1(1.67 \%)$ & $12(20 \%)$ & $13(21.67 \%)$ \\
\hline Stage-IV & $4(6.67 \%)$ & $25(41.67 \%)$ & $29(48.33 \%)$ \\
\hline Total & $8(13.33 \%)$ & $52(86.67 \%)$ & $60(100 \%)$ \\
\hline
\end{tabular}

\section{TNM STAGING}
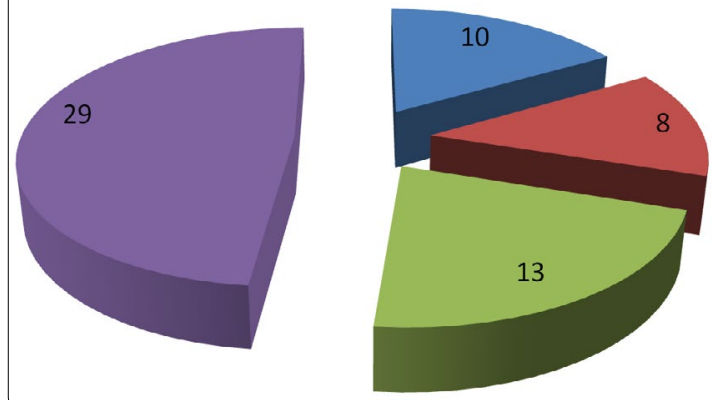

STAGE 1

- STAGE 2

- STAGE 3

- STAGE 4

Graph 1. Clinical Stage
All patients with ulcer abutting lower gingivobuccal sulcus subjected to X-Ray mandible. Orthopantomogram(OPG) done in 26(43.33\%) of cases. 37(61.67\%) cases clinically shows positive nodes, $23(38.33 \%)$ cases shows clinically negative nodes. 37(61.67\%) cases underwent Modified radical neck dissection(MRND). 13(21.67\%) underwent supra-omohyoid neck dissection(SOHND), 10(16.67\%) cases in Stage-I not subjected to neck dissection ( $p<0.001$ ) (Table 2 and Graph 2). $48(80 \%)$ of patients underwent mandibulectomy, no mandibulectomy in 12(20\%) ( $p=0.548)$ (Table 3 and Graph 3).

Table 2. Type of Neck Dissection

\begin{tabular}{|c|c|c|c|}
\hline Neck Dissection & Male & Female & Total \\
\hline MRND & $4(6.67 \%)$ & $33(55 \%)$ & $37(61.67 \%)$ \\
\hline SOHND & $3(5 \%)$ & $10(16.67 \%)$ & $13(21.67 \%)$ \\
\hline No Neck Dissection & $1(1.67 \%)$ & $9(15 \%)$ & $10(16.67 \%)$ \\
\hline Total & $8(13.33 \%)$ & $52(86.67 \%)$ & $60(100 \%)$ \\
\hline
\end{tabular}

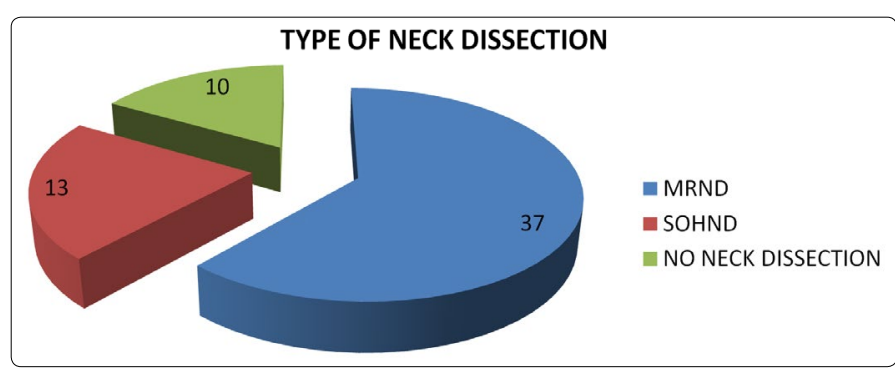

Graph 2. Type of Neck Dissection

Table 3. Type of Mandibulectomy

\begin{tabular}{|c|c|c|c|}
\hline Mandibulectomy & Male & Female & Total \\
\hline Hemi-mandibulectomy & $5(8.33 \%)$ & $37(61.67 \%)$ & $42(70 \%)$ \\
\hline Marginal mandibulectomy & $1(1.67 \%)$ & $3(5 \%)$ & $4(6.67 \%)$ \\
\hline Segmental mandibulectomy & -- & $2(3.33 \%)$ & $2(3.33 \%)$ \\
\hline No mandibulectomy & $2(3.33 \%)$ & $10(16.67 \%)$ & $12(20 \%)$ \\
\hline Total & $8(13.33 \%)$ & $52(86.67 \%)$ & $60(100 \%)$ \\
\hline
\end{tabular}

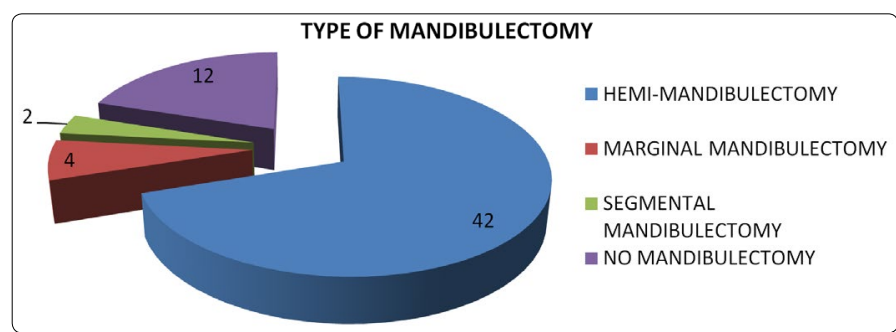

Graph 3. Type of Mandibulectomy

$15(25 \%)$ and $17(28.33 \%)$ of patients underwent reconstruction with the Pectoralis major myocutaneous flap (PMMC) \& PMMC+DP Flaps respectively. 6(10\%) patients underwent tongue flap while $5(8.33 \%)$ patients underwent forehead flap. 4(6.67\%) and $1(1.67 \%)$ patients underwent Deltopectoral(DP) and toungue+DP flap respectively ( $p$ <0.0001) (Table 4 and Graph 4).

Table 4. Type of Reconstruction

\begin{tabular}{|c|c|c|c|}
\hline Flaps & Male & Female & Total \\
\hline PMMC & $2(3.33 \%)$ & $13(21.67 \%)$ & $15(25 \%)$ \\
\hline PMMC+DP & $2(3.33 \%)$ & $15(25 \%)$ & $17(28.33 \%)$ \\
\hline Tongue & $1(1.67 \%)$ & $5(8.33 \%)$ & $6(10 \%)$ \\
\hline Forehead & $1(1.67 \%)$ & $4(6.67 \%)$ & $5(8.33 \%)$ \\
\hline DP & -- & $4(6.67 \%)$ & $4(6.67 \%)$ \\
\hline Tongue + DP & -- & $1(1.67 \%)$ & $1(1.67 \%)$ \\
\hline SSG & -- & $7(11.67 \%)$ & $7(11.67 \%)$ \\
\hline No Reconstruction & $2(3.33 \%)$ & $3(5 \%)$ & $5(8.33 \%)$ \\
\hline Total & $8(13.33 \%)$ & $52(86.67 \%)$ & $60(100 \%)$ \\
\hline
\end{tabular}




\section{TYPE OF RECONSTRUCTION}
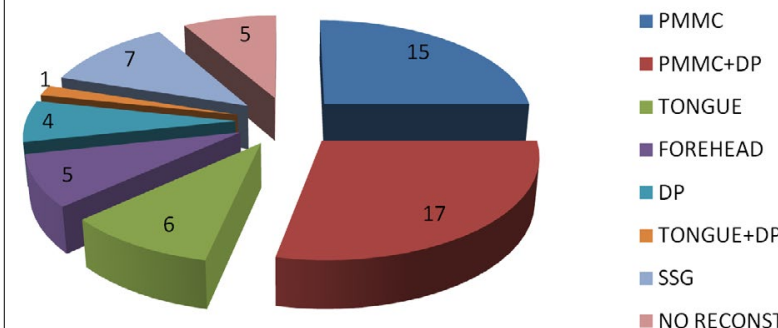

NORECONSTRUCTION

Graph 4. Type of Reconstruction

42(70\%) patients in Stage-III and Stage-IV received Radiotherapy(RT)+Chemotherapy(CT), 18(30\%) patients were in Stage-I and Stage-II of which 8(13.33\%) patients in Stage-I not received RT, while 2(3.33\%) cases lost follow-up ( $p=0.02$ ). Hence, 8(13.33\%) patients received RT and 10(16.67\%) patients didn't receive RT ( $p=0.02$ ) (Table 5 and Graph 5).

Table 5. Radiotherapy and Chemotherapy

\begin{tabular}{|c|c|c|c|}
\hline Treatment & Male & Female & Total \\
\hline Radiotherapy & $3(5 \%)$ & $5(8.33 \%)$ & $8(13.33 \%)$ \\
\hline Radiotherapy+Chemotherapy & $4(6.67 \%)$ & $38(63.33 \%)$ & $42(70 \%)$ \\
\hline No Radiotherapy & $1(1.67 \%)$ & $9(15 \%)$ & $10(16.67 \%)$ \\
\hline Total & $8(13.33 \%)$ & $52(86.67 \%)$ & $60(100 \%)$ \\
\hline
\end{tabular}

RADIOTHERAPY AND CHEMOTHERAPY

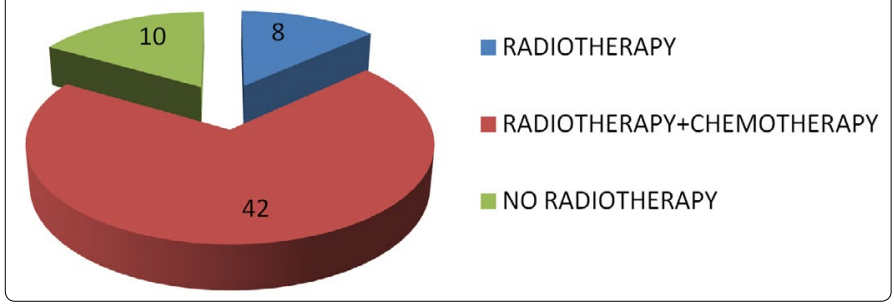

Graph 5. Radiotherapy and Chemotherapy

Postoperative complications like infection and necrosis occurred in $3(5 \%)$ and $1(1.67 \%)$ cases respectively, while orocutaneous fistula occurred in $1(1.67 \%)$ cases and $1(1.67 \%)$ case showed recurrence.

\section{Discussion}

Cancers of the oral cavity account for $3-4 \%$ of all malignancies. The high incidence of oral cancers in our country has been attributed to the peculiar social habits which include chewing tobacco, betal nut or placing a quid either in the posterior half of gingivobuccal sulcus or in the gingivolabial sulcus. In the Western countries, smoking and alcohol are the major causative agents. In our country, Sanghavi et al [11] have demonstrated the association of bidi smoking in addition to paan chewing with oral cancer. Further they have been shown to have a synergistic effect and it was found that about $81.67 \%$ of the patients indulged in chewing tobacco while $6.67 \%$ indulged in smoking and tobacco consumption, and $11.67 \%$ indulged in smoking, alcohol \& tobacco consumption.

In this study; mean age group involved was $52.63 \pm 7.48$ years, highest incidence seen in $50-59$ yrs with females $86.67 \%$ predominating over males $13.33 \%$ ( $p<0.001$ ), where in the literature it is the males predominating the females, highest incidence seen between $4^{\text {th }}$ - $5^{\text {th }}$ decades.

The majority of patients when seen first belonged to StageIV (48.33\%) and Stage-I \& II have $16.67 \%$ and $13.33 \%$ respectively, while Stage III was $21.67 \%$ respectively ( $p=0.003$ ). The lack of health consciousness, the low socio-economic status, illiteracy, the peculiar social habits, missed early lesions and the paucity of well equipped centers account to a large extent in the delay with which these patients present. $73.33 \%$ patients presented with a non-healing ulcer in the buccal mucosa, $6.67 \%$ presents with the swelling \& ulcer, $20 \%$ presents with ulcer $\&$ trismus $(p=0.126)$.

Approximately (60\%) of patients had extensive disease beyond the cheek mucosa. This is comparable with the study done by Martin and P flueger (1935) [12], and O'Brien and Catlin (1965) [13].

Out of the 60 cases, 37(61.67\%) cases clinically shows positive nodes, 23(38.33\%) cases shows clinically negative nodes. 37(61.67\%) cases underwent MRND. 13(21.67\%) underwent SOHND, 10(16.67\%) cases in Stage-I not subjected to neck dissection ( $p$ <0.001). According to Bloom; Spiro and Weng [16], an elective neck dissection may be omitted in patients with early lesions.

According to Maccomb Fletcher [14] and O'Brien Catlin [13], in advanced stages of disease and clinically negative neck, the wide resection of the primary tumor should be combined with neck dissection, as the percentage of occult metastases in the neck is about (30\%), for the oral cavity cancer. The issue is controversial.

In the management of metastatic cervical lymph nodes most authors agree that a radical neck dissection had to be done. In a recent study, it was found that a radical neck dissection is indeed superior to radiation therapy in treating metastatic lymph nodes. 42(70\%) hemi-mandibulectomies, 4(6.67\%) marginal mandibulectomies \& 2(3.33\%) segmental mandibulectomies were performed in the present study ( $p=0.548$ ). Mandibulectomy was not done in $12(20 \%)$ patients. In the past hemi-mandibulectomies were performed even for early lesions, since it was believed that the periosteal lymphatics contains tumor emboli but it has been proved that spread to the mandible is governed by the proximity of the tumor rather than the periosteal lymphatics.

$15(25 \%)$ and $17(28.33 \%)$ of patients underwent reconstruction with the PMMC \& PMMC+DP Flaps respectively. It tolerated well following radiation therapy. In immediate postoperative period not accepted by patients because it gives bulkiness over face, pulling sensation in the neck, later in terms of oral competence, speech swallowing accepted by all patients. 4(6.67\%) and 1(1.67\%) patients underwent DP and tongue +DP flap respectively. 6(10\%) patients underwent tongue flap while 5(8.33\%) patients underwent forehead flap. Tongue flap is a single stage primary repair with short hospitalization, it is a rapid procedure and time required for procedure is also short, with simple technique. Flaps remain viable because of rich vascularity of the tongue, even twist at the pedicle also harmless. All patients with tongue flaps are not 
satisfied in terms of speech, oral competence \& swallowing. DP flaps requires a 2 staged procedure, cosmetically patients were not happy. Forehead flap patients were unhappy because recipient area requires SSG \& there will be loss off rowning in the forehead \& cosmetically were not acceptable by patients.

$42(70 \%)$ patients in Stage-III and Stage-IV received $\mathrm{RT}+\mathrm{CT}$, $18(30 \%)$ patients were in Stage-I and Stage-II of which 8(13.33\%) patients in Stage-I not received RT, while 2(3.33\%) cases lost follow-up. Hence, 8(13.33\%) patients received RT and 10(16.67\%) patients didn't receive RT. Studies by Huang et al [15] suggested that post-operative radiation therapy offers a significant benefit in terms of loco-regional control and survival in patients with head and neck carcinoma.

The loco-regional recurrence rate in this study was found to be $1.67 \%$, In this study, low loco-regional recurrence rate is due to patients treated with surgery followed by RT and combined with $\mathrm{CT}$ in advanced stages. This confirms the fact that post-operative radiotherapy gives a good loco-regional control. The loco-regional rate in the literature is $37-45 \%$ comparable with the study conducted by Kim I et al [16]. The loco-regional recurrences occurred in 1 (1.67\%) patient, 3 months after surgery followed by 2 cycles of RT, remaining cycles of RT was not received \& presented with recurrence \& underwent reconstruction with PMMC + DP flap followed by $\mathrm{RT}+\mathrm{CT}$. The total complication rate $(8.33 \%)$ was seen to be the low incidence of which compares well with other studies. These included infection, necrosis in $3(5 \%)$ and $1(1.67 \%)$ cases respectively, while oro-cutaneous fistula occurred in $1(1.67 \%)$ cases. These were managed by regular debridement \& converting oral fistula into oro-cutaneous fistula. Highest incidence is seen in PMMC \& combination flaps.

\section{Conclusion}

Patients subjected to reconstruction of cheek defect with PMMC and PMMC + DP flap shows better results in terms of oral competence, speech, swallowing \& cosmetic appearance when compared to other flaps. Tongue flap shows poor results in terms of oral competence, speech \& swallowing, there was a patch retraction with salivary leakage in $2(3.33 \%)$ patients. Patients with the forehead flap give good functional results with poor cosmetic appearance. In our overall experiences PMMC alone and PMMC with DP flap gives better results both functionally and cosmetically.

\section{Compliance with Ethical Standards}

Informed consents were taken from all participants, and the study protocol was approved by Institutional ethical committee approval board.

\section{Conflict of interest}

The authors confirm that there is no conflict of interest regarding this manuscript.

\section{References}

1. Bailey \& Love's Short practice of surgery $25^{\text {th }}$ edition pg 734-738. Batsakis JG: Tumors of the head and neck. Pg 144-65.

2. Kasturi J. Perin MN. Epidemiology of oral cancer. Oral Cancer: 1991: 1-8. Bloom ND et al. Carcinoma of the cheek mucus. Am J Surg. 1980; 140: 556-55.

3. Parkin DM, Bray F, Ferlay J, Pisani P. Estimating the world cancer burden: Globocan 2000. Int J Cancer. 2001; 94(2): 153-156.

4. National Cancer Registry Programme: Consolidated report of Hospital Based Registries. 1994-1998.

5. Pradhan SA. Surgery for the cancer of the buccal mucosa. Semin Surg Oncol. 1989; 5(5): 318-21.

6. Iyer SG, Pradhan SA, Pai PS. Surgical treatment outcomes of localized squamous carcinoma of buccal mucosa. Head Neck. 2004; 26(10): 897902.

7. O'Brien CJ, Nettle WJ, Lee KK. Changing trends in the management of carcinoma of the oral cavity and oropharynx. Aust NZ J Surg. 1993; 63(4): 270-274. doi: 10.1111/j.1445-2197.1993.tb00381.x

8. Fang FM, Leung SW, Huang CC, Liu YT, Wang CJ, et al. Combined modality therapy for squamous carcinoma of the buccal mucosa: treatment results and prognostic factors. Head Neck. 1997; 19(6): 506-512. doi: 10.1002/ (SICI)1097-0347(199709)19:6<506::AID-HED8>3.0.CO;2-2

9. Berizh, Strauch et al. Grabb's and Smith's plastic surgery 6th edition. Head Neck. 2007.

10. Manni JJ, van den Hoogen FJ. Supraomohyoid neck dissection with frozen section biopsy as a staging procedure in the clinically node negative neck in carcinoma of the oral cavity. Am J Surg. 1991; 162(4): 373-376. doi: 10.1016/0002-9610(91)90151-3

11. Sanghavi et al: Tobacco and Cancer. British Med J. 1955: 91: 1111-1114.

12. Martin $\mathrm{HE}$, Pflueger $\mathrm{OH}$. Cancer of the cheek (buccal mucosa) Study of ninety-nine cases with results of treatment at the end of five years. Arch surg. 1935; 30(5): 731-747. doi:10.1001/archsurg.1935.01180110002001

13. O'Brien, PH, Catlin D. Cancer of the cheek (mucosa). Cancer. 1965; 18(11): 1392-1398. doi: 10.1002/1097-0142(196511)18:11<1392::AIDCNCR2820181103>3.0.CO;2-E.

14. Vegers JWM, Snow GB, van der Waal I. Squamous Cell Carcinoma of the Buccal Mucosa A Review of 85 Cases. Arch Otolaryngol. 1979; 105(4): 192195. doi:10.1001/archotol.1979.00790160026006

15. Huang CH, Chu ST, Luo-Ping G, Hou YY, et al. Clinicopathologic Evaluation of Prognostic Factors for Squamous Cell Carcinoma of the Buccal Mucosa. J Chinese Med Assoc. 2007; 70(4): 164-170. doi: 10.1016/S17264901(09)70351-X

16. Kim I, Myoung $H$. Squamous cell carcinoma of the buccal mucosa involving the masticator space: a case report. J Korean Assoc Oral Maxillofac Surg. 2017; 43(3): 191-196. doi:10.5125/jkaoms.2017.43.3.191 